\title{
Détermination indirecte des coefficients de diffusion de la vapeur d'eau dans les directions tangentielle et radiale du bois de hêtre
}

\author{
Nadia Mouchot, André Wehrer, Voichita Bucur and André Zoulalian* \\ LERMAB-ENSTIB, Université Henri Poincaré Nancy I, B.P 239, 54506 Vandœuvre-lès-Nancy, France
}

(Reçu le 2 janvier 1999; accepté le 31 mars 2000)

\begin{abstract}
Résumé - Afin de caractériser le transport diffusionnel de l'eau dans le domaine hygroscopique du bois, nous proposons d'estimer initialement les coefficients de diffusion de la vapeur d'eau dans les trois directions orthotropiques du bois. Les coefficients de diffusion de la vapeur d'eau sont déduits des coefficients de diffusion d'un soluté inerte (hélium) mesurés dans une cellule de Wicke et Kallenbach évoluant en système transitoire. Les coefficients de diffusion dans les directions radiales et tangentielles mesurés à $30^{\circ} \mathrm{C}$ sont indépendants de l'humidité absolue du bois. Les valeurs trouvées sont compatibles avec les intervalles de variations donnés dans la littérature, le coefficient de diffusion radial du bois de hêtre étant sensiblement plus élevé que le coefficient de diffusion tangentiel, probablement par la présence de rayons ligneux en direction radiale.
\end{abstract}

coefficient de diffusion / domaine hygroscopique / hêtre / diffusivité vapeur d'eau / cellule de diffusion Wicke et Kallenbach

\begin{abstract}
Indirect determination of tangential and radial steam diffusitivity of beech. In order to characterize the diffusion process of water in the hygroscopic range of wood, we propose to evaluate initially the diffusion coefficients of water vapour in the three orthotropic directions of wood. The diffusion coefficients of water vapour are deduced from the diffusion coefficients of an inert solute (helium) measured in a diffusion cell, type Wicke and Kallenbach, being in an unsteady state. The diffusion coefficients in the radial and tangential directions measured at $30{ }^{\circ} \mathrm{C}$ are independent of absolute moisture content of wood. The values we find agree with ranges of variations given in the literature. The diffusion coefficient in the radial direction is roughly higher than the diffusion coefficient in the tangential direction, probably due to the rays in the radial direction.
\end{abstract}

diffusion coefficient / hygroscopic range / beech / steam diffusivity / Wicke and Kallenbach diffusion cell

\section{Nomenclature}

$C$ : concentration d'eau vapeur $\left(\mathrm{mol} \mathrm{m}^{-3}\right)$

$C^{\prime}$ : concentration d'eau liée $\left(\mathrm{mol} \mathrm{m}^{-3}\right)$

$C_{\mathrm{I}}$ : concentration en inerte $\left(\mathrm{mol} \mathrm{m}^{-3}\right)$

$C_{1}(0)$ et $C_{2}(0)$ : concentrations initiales en hélium dans les compartiments 1 et $2\left(\mathrm{~mol} \mathrm{~m}^{-3}\right)$
$C_{1}$ et $C_{2}$ : concentrations en hélium dans les compartiments 1 et $2\left(\mathrm{~mol} \mathrm{~m}^{-3}\right)$

$D_{I}$ : coefficient de diffusion de l'inerte dans le bois $\left(\mathrm{m}^{2} \mathrm{~s}^{-1}\right)$

$D$ : coefficient de diffusion de l'eau vapeur dans le bois $\left(\mathrm{m}^{2} \mathrm{~s}^{-1}\right)$

$\mathbb{D}_{\mathrm{I}_{2}}$ : coefficient de diffusion moléculaire de l'inerte $\left(\mathrm{m}^{2} \mathrm{~s}^{-1}\right)$

* Correspondance et tirés-à-part

Tél. (33) 03839120 57; Fax. (33) 03839121 02; e-mail : andre.zoulalian@lermab.u-nancy.fr 
$\mathbb{D}$ : coefficient de diffusion moléculaire de l'eau vapeur $\left(\mathrm{m}^{2} \mathrm{~s}^{-1}\right)$

$\varepsilon$ : porosité du bois (-)

$e$ : épaisseur de l'échantillon $(\mathrm{m})$

$K S$ : conductance globale de transfert $\left(\mathrm{m}^{3} \mathrm{~s}^{-1}\right)$

$R_{e}$ : résistance diffusionnelle des deux couches limites $\left(\mathrm{m}^{-3} \mathrm{~s}^{-1}\right)$

$R_{i}$ : résistance diffusionnelle de l'échantillon $\left(\mathrm{m}^{-3} \mathrm{~s}^{-1}\right)$

$S$ : surface de l'échantillon $\left(\mathrm{m}^{2}\right)$

$\tau:$ temps (s)

$T$ : température $(\mathrm{K})$

$V_{1}$ et $V_{2}$ : volumes des compartiments 1 et $2\left(\mathrm{~m}^{3}\right)$

\section{Indices}

1 : dans la cellule 1

2 : dans la cellule 2

$\mathrm{I}$ : inerte

\section{INTRODUCTION}

Dans le domaine hygroscopique du bois (humidité absolue du bois inférieure au point de saturation des fibres), il existe entre la teneur en eau du bois et la teneur en eau de la phase gazeuse environnante une relation d'équilibre dépendant de la température. Ainsi, lors du séchage, l'humidité absolue pour le bois (teneur en eau base sèche) correspondra à l'état d'équilibre avec le milieu extérieur. De plus, dans le domaine hygroscopique, le séchage a besoin d'être contrôlé car en l'absence d'efforts extérieurs, les contraintes internes liées au retrait du bois peuvent atteindre des niveaux très élevés entraînant fissuration, gauchissement et courbure du bois.

Dans le domaine hygroscopique et à température modérée (inférieure à $80^{\circ} \mathrm{C}$ ) les transports de matière et d'énergie au sein du bois sont respectivement diffusif pour l'eau et conductif pour l'énergie thermique. L'évaluation des densités de flux de matière et d'énergie nécessitent alors la connaissance de coefficients de diffusion et de conductivités thermiques.

L'objectif final de notre travail est de proposer une nouvelle approche pour la modélisation du transport diffusionnel de l'eau dans le domaine hygroscopique prenant en compte le caractère anisotropique du bois et l'influence de la teneur en eau sur la structure interne et les propriétés physiques du bois humide.

L'application des lois de Fick en régime stationnaire et transitoire a été utilisée ces dernières années par différents auteurs pour une évaluation du coefficient de diffusion de l'eau liée et vapeur au sein du bois. On peut citer en particulier les travaux de $[2,4,5,6,7,8]$.
Pour une même essence, les mesures stationnaires et transitoires n'aboutissent généralement pas aux mêmes valeurs de coefficients de diffusion dans la mesure où les gradients considérés dans l'application des lois de Fick sont différents (gradient de concentration en phase vapeur, gradient de concentration d'eau liée, gradient de potentiel chimique, etc.). De plus, la dispersion des résultats est due également à la nature des essences, à la variabilité tant au niveau de l'essence que des échantillons sur lesquels sont effectuées les mesures.

Afin de quantifier au mieux le transport diffusionnel de l'eau (eau vapeur et eau liée) au sein du bois, nous proposons d'utiliser une méthodologie appliquée avec succès dans le cas du transport diffusionnel de solutés gazeux adsorbables dans un support solide macro et microporeux comme les catalyseurs solides. Nous présentons ci-après les bases théoriques de cette approche qui pourra être utilisée après validation expérimentale pour représenter le transport diffusionnel de l'eau dans le domaine hygroscopique du bois.

\section{BASES THÉORIQUES POUR L'ÉVALUATION DU TRANSPORT DIFFUSIONNEL DE L'EAU DANS LE DOMAINE HYGROSCOPIQUE}

\subsection{Modélisation du transport diffusionnel d'un soluté adsorbable au sein d'un milieu poreux}

Soit un milieu poreux en présence d'un soluté gazeux adsorbable (figure 1).

Si $C$ et $C$ ' désignent les concentrations en soluté respectivement en phase gaz et en phase adsorbée, le transport du soluté dans le solide fait apparaître trois étapes, soit :

* un transport diffusionnel en phase gazeuse caractérisé par un coefficient de diffusion $D$;

* un transport diffusionnel en phase adsorbée caractérisé par un coefficient de diffusion $D^{\prime}$;

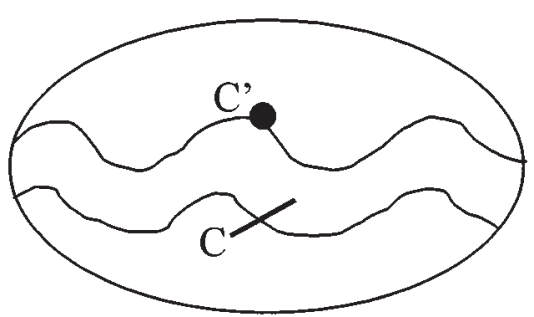

Figure 1. Représentation schématique d'un milieu poreux. 
* un échange entre le soluté en phase gazeuse et le soluté adsorbé dont l'état limite (absence de résistance au transfert) correspond à l'équilibre thermodynamique.

Expérimentalement on ne peut pas déterminer la contribution de chacune des trois étapes à partir d'une information qui est toujours globale.

C'est pourquoi, la modélisation suggérée est basée sur les hypothèses simplificatrices suivantes :

* l'échange entre le soluté en phase gazeuse et le soluté adsorbé n'est pas limité par des résistances de transfert. Dans ces conditions, l'équilibre thermodynamique est observé, ce qui se traduit par une relation entre les concentrations $C$ et $C$ ' qui peut être obtenue expérimentalement dans des expériences adaptées. Cette relation dépend généralement de la température. Elle est du type :

$$
C^{\prime}=\mathrm{f}(C, T) \text {. }
$$

* le transport diffusionnel en phase adsorbée est supposé très faible vis-à-vis du transport diffusionnel en phase gazeuse. Dans ces conditions, le bilan de matière local du soluté au sein du milieu poreux s'écrit :

$$
\varepsilon \Delta(D C)-\varepsilon \frac{\partial C}{\partial \tau}-(1-\varepsilon) \frac{\partial C^{\prime}}{\partial \tau}=0
$$

Dans la relation (2), $\varepsilon$ est la porosité du milieu poreux. $\Delta$ représente l'opérateur laplacien vis-à-vis des variables d'espace, $\tau$ est le temps.

La modélisation du transport diffusionnel du soluté s'obtient par résolution des équations (1) et (2) auxquelles il faudra adjoindre les équations de bilans d'énergie dans le cas où le transport du soluté est associé à un transport d'énergie thermique.

Dans le cas particulier d'un fonctionnement isotherme, la connaissance de l'équilibre d'adsorption, de la porosité du milieu et du coefficient de diffusion $D$ sont suffisants pour évaluer le flux de soluté transféré au sein du milieu poreux. Inversement, la connaissance du flux, de l'équilibre d'adsorption et de la porosité permet d'obtenir le ou les coefficients de diffusion (milieu solide non isotrope). C'est cette méthodologie que nous proposons de vérifier pour caractériser le transport diffusionnel de l'eau dans le domaine hygroscopique du bois de hêtre. Pour sa mise en œuvre, il est nécessaire de connaître le coefficient de diffusion de l'eau vapeur au sein du bois. Toutefois, cette caractéristique de transport ne peut pas être atteinte directement dans le cas de la vapeur d'eau car l'eau vapeur s'adsorbe sur le bois et conduit à un gonflement du bois et donc à une modification de la porosité.
Pour lever cette difficulté tout en prenant en compte les modifications de la géométrie, nous proposons de mesurer le coefficient de diffusion d'un soluté gazeux inerte (non adsorbable) au sein du bois dont la teneur en eau est fixée et en équilibre avec la teneur en eau de la phase gazeuse inerte. Pour ce soluté inerte, l'équation de bilan se réduit à :

$$
\Delta\left(D_{\mathrm{I}} C_{\mathrm{I}}\right)-\frac{\partial C_{\mathrm{I}}}{\partial \tau}=0
$$

\subsection{Estimation du coefficient de diffusion de la vapeur d'eau}

Connaissant le coefficient $D_{\mathrm{I}}$ dans une direction donnée, le coefficient de diffusion de la vapeur d'eau $D$ s'obtient alors en admettant que les coefficients de diffusion dans le milieu poreux sont proportionnels aux coefficients de diffusion moléculaire, soit :

$$
\frac{D}{D_{\mathrm{I}}}=\frac{\mathbb{D}}{\mathbb{D}_{\mathrm{I}}}
$$

$\mathbb{D}$ et $\mathbb{D}_{\mathrm{I}}$ représentent respectivement les coefficients de diffusion moléculaire de la vapeur d'eau et de l'inerte dans la phase gazeuse.

Il est possible d'évaluer ces coefficients à partir des relations déduites de la théorie cinétique des gaz ou des relations semi-empiriques déduites de la littérature dont les principales sont données dans l'ouvrage de Reid et coll. [9]

Dans ce qui suit, nous limiterons les mesures expérimentales à la détermination des coefficients de diffusion dans les directions radiales et tangentielles du bois de hêtre pour un soluté inerte, à savoir l'hélium. Nous en déduirons les valeurs du coefficient de diffusion de l'eau en phase vapeur dans le bois de hêtre dans les deux directions précitées.

\section{DÉTERMINATION DES COEFFICIENTS DE DIFFUSION D'UN SOLUTÉ INERTE DANS DU BOIS DE HÊTRE DONT L'HUMIDITÉ ABSOLUE CORRESPOND AU DOMAINE HYGROSCOPIQUE}

\subsection{Description du dispositif expérimental}

Les mesures du coefficient de diffusion sont réalisées dans une cellule classique de diffusion du type Wicke et Kallenbach (figure 2) où les deux compartiments sont séparés par l'échantillon pour lequel on cherche à 
a) schéma :

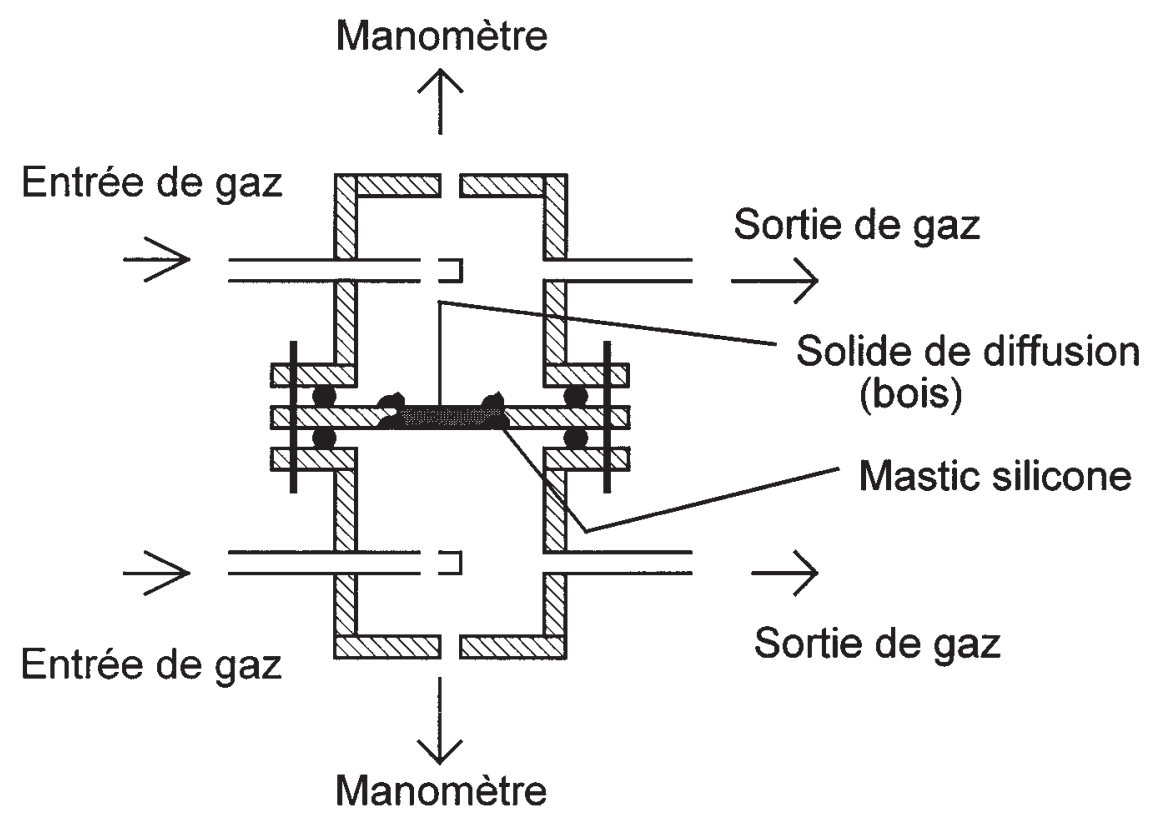

b) photographie de la cellule :

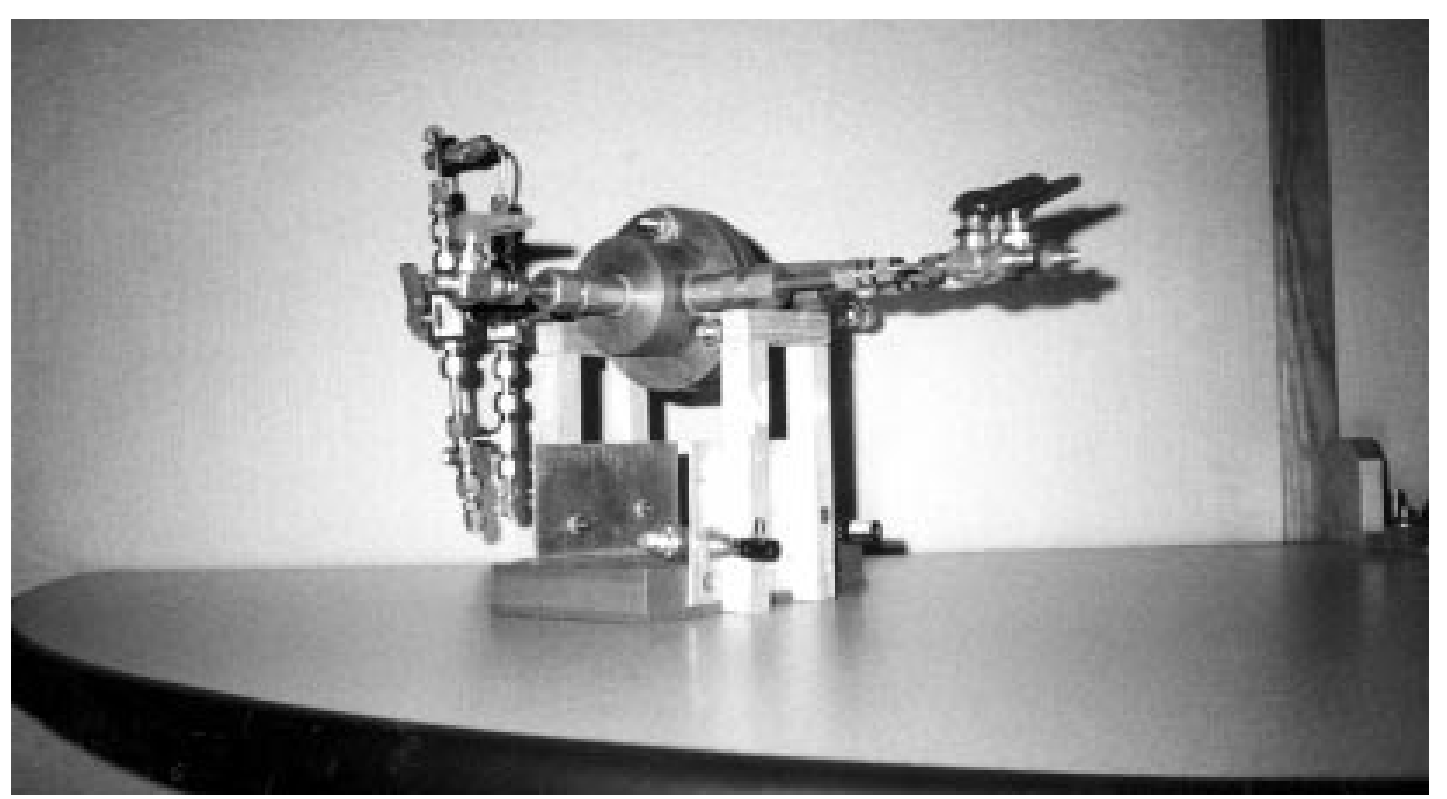

Figure 2. Cellule de diffusion du type Wicke-Kallenbach. 
prévoir le coefficient de diffusion. Les deux compartiments cylindriques de diamètre intérieur $40 \mathrm{~mm}$ ont en l'absence d'échantillon un volume identique de $63 \mathrm{~cm}^{3}$.

L'échantillon de bois est maintenu au centre d'une rondelle métallique séparant les deux compartiments à l'aide d'une colle de type silicone suffisamment élastique pour supporter les variations dimensionnelles de l'échantillon tout en assurant une bonne étanchéité. Nous avons vérifié que la perméabilité et la diffusion de l'hélium et de l'azote étaient négligeables devant les phénomènes observés en présence des échantillons de bois. L'étanchéité entre la rondelle et les deux compartiments est obtenue par l'intermédiaire de joints et de vis de serrage rendant solidaires les deux compartiments.

Les compartiments sont traversés par des gaz inertes de nature différente (hélium pour le compartiment 1 , azote pour le compartiment 2). L'alimentation des gaz se fait par des tubes de diamètre intérieur $4 \mathrm{~mm}$ percés de 1 à 3 orifices de diamètre inférieur à $1 \mathrm{~mm}$ afin d'assurer une bonne homogénéité de la phase gazeuse au sein du compartiment lors d'un fonctionnement du compartiment en système ouvert. Les deux courants gazeux peuvent être humidifiés à une humidité donnée en faisant traverser les gaz dans des barboteurs contenant de l'eau à une température inférieure ou égale à la température de fonctionnement de la cellule fixée à $30{ }^{\circ} \mathrm{C}$. Dans ces conditions, l'échantillon de bois atteint une humidité absolue en équilibre avec les humidités des phases gazeuses qui sont identiques dans la mesure où la température des deux barboteurs est similaire.

Les mesures du coefficient de diffusion sont effectuées sur une seule essence, à savoir le hêtre. Tous les échantillons ont été découpés dans un même avivé d'épaisseur $50 \mathrm{~mm}$ en choisissant des directions normales aux trois directions orthotropiques. Les mesures expérimentales rapportées ci-après concernent seulement des échantillons dont la surface est normale aux directions tangentielle et radiale. Les épaisseurs des échantillons sont comprises entre 1 et $4 \mathrm{~mm}$. Les mesures sont effectuées sur des disques de $30 \mathrm{~mm}$ dont la surface non recouverte par la colle à base de silicone est $S=5,73 \mathrm{~cm}^{2}$.

Les mesures de diffusivité au niveau de la cellule peuvent être réalisées en régime stationnaire ou en régime transitoire. Dans les deux cas, le transport des solutés au sein des échantillons de bois se limite à un transport purement diffusionnel si et seulement si la différence de pression totale entre les deux compartiments est nulle. Expérimentalement, celle-ci reste inférieure à $1 \mathrm{~mm}$ de colonne d'eau. La perméabilité axiale du hêtre étant très supérieure aux perméabilités radiale et tangentielle, nous limitons les mesures aux directions tangentielle et radiale dans la mesure où nous pouvons supposer que le flux convectif dans l'échantillon est faible devant le flux dif- fusionnel, lui-même petit devant les flux traversant les deux compartiments. Dans ces conditions, le fonctionnement stationnaire de la cellule devient peu exploitable (les concentrations en hélium dans le courant d'azote et en azote dans le courant d'hélium sont faibles) et seule l'étude transitoire de la cellule peut être envisagée pour l'évaluation du flux diffusif et par suite du coefficient de diffusion. Nous précisons ci-après la modélisation de la cellule dans le cas d'un fonctionnement transitoire.

\subsection{Modélisation de la cellule de diffusion en régime transitoire}

Suite à un fonctionnement stationnaire de la cellule, on coupe à un instant donné pris comme origine du temps $(\tau=0)$ les débits d'alimentation et d'évacuation des deux compartiments. À partir de cet instant les deux cellules vont donc évoluer en système fermé et un transfert diffusionnel va apparaître entre les deux compartiments. $C_{1}$ et $C_{2}$ désignant les concentrations d'hélium à l'instant $\tau$ au niveau des compartiments 1 et 2 , les bilans sur l'ensemble de la cellule et dans le compartiment 1 de l'hélium s'écrivent respectivement :

$$
\begin{gathered}
V_{1} C_{1}+V_{2} C_{2}=V_{1} C_{1}(0)+V_{2} C_{2}(0) \\
-V_{1} \frac{\mathrm{d} C_{1}}{\mathrm{~d} \tau}=K S\left(C_{1}-C_{2}\right) .
\end{gathered}
$$

Dans les équations (5) et (6), $V_{1}$ et $V_{2}$ représentent les volumes respectifs des compartiments 1 et 2 . $C_{1}(0)$ et $C_{2}(0)$ désignent les concentrations d'hélium dans les compartiments 1 et 2 à l'instant initial. $S$ est l'aire de surface plane de l'échantillon en contact avec la phase gazeuse au niveau de chaque compartiment. $K$ représente un coefficient global de transfert de matière qui tient compte des transports diffusionnels dans la couche limite au niveau des deux compartiments et au sein de l'échantillon de bois.

La résolution des équations de bilan en tenant compte des valeurs initiales de $C_{1}$ et $C_{2}$, conduit aux relations :

$$
\begin{aligned}
& -\operatorname{Ln}\left[\frac{V_{1} C_{1}(0)+V_{2} C_{2}(0)-\left(V_{1}+V_{2}\right) C_{1}}{V_{2}\left(C_{2}(0)-C_{1}(0)\right)}\right]=-\operatorname{Ln}\left[A_{1}\right]=\frac{K S\left(V_{1}-V_{2}\right)}{V_{1} V_{2}} \tau \\
& -\operatorname{Ln}\left[\frac{V_{1} C_{1}(0)+V_{2} C_{2}(0)-\left(V_{1}+V_{2}\right) C_{2}}{V_{1}\left(C_{1}(0)-C_{2}(0)\right)}\right]=-\operatorname{Ln}\left[A_{2}\right]=\frac{K S\left(V_{1}+V_{2}\right)}{V_{1} V_{2}} \tau .
\end{aligned}
$$

Les relations précédentes montrent que la variation des deux premiers membres en fonction du temps est une 
droite dont la pente est directement liée à la conductance globale $K S$. On peut donc valider les relations précédentes sur toute la durée des essais et donc avoir une plus grande précision sur la valeur de la conductance $K S$.

D'une manière générale, l'inverse de la conductance globale représente la résistance globale de transfert. Cette résistance globale est la somme de la résistance diffusionnelle dans l'échantillon $R_{i}$ et de la résistance diffusionnelle des deux couches limites $R_{e}$, d'où :

$$
\frac{1}{K S}=R_{i}+R_{e}
$$

la résistance diffusionnelle dans l'échantillon est liée à l'épaisseur $e$ de l'échantillon et au coefficient de diffusion $D_{\mathrm{I}}$, soit :

$$
R_{i}=\frac{e}{D_{\mathrm{I}} S} .
$$

À partir des valeurs de la conductance de transfert $K S$ pour différentes épaisseurs d'échantillons, l'application de la relation (10) va permettre une évaluation du coefficient de diffusion $D_{\mathrm{I}}$. Les différentes valeurs de $K S$ étant obtenues sur des échantillons indépendants, la valeur moyenne obtenue pour $D_{\text {I }}$ prend partiellement en compte l'incertitude des mesures expérimentales et la variabilité des échantillons au niveau d'un même avivé. Une estimation plus précise du domaine de variation du coefficient de diffusion lié à la variabilité de l'échantillon nécessiterait, pour chaque épaisseur d'échantillon, des mesures transitoires sur une dizaine d'échantillons. À chaque temps d'opération, la mesure doit être reproduite deux fois, l'analyse de la concentration en hélium ne pouvant être effectuée que dans un compartiment. En définitive, chaque expérience transitoire se déroulant sur plus de six heures, le nombre d'échantillons examinés est resté limité. Toutefois, nous avons vérifié que les mesures sur un même échantillon étaient globalement bien reproductibles.

Les concentrations d'hélium au niveau des deux compartiments sont obtenues par l'intermédiaire d'un détecteur catharométrique étalonné associé à une boucle d'échantillonnage dont le volume est connu. L'indication du catharomètre et la courbe d'étalonnage permettent de remonter à la concentration d'hélium dans la boucle puis à celle du compartiment analysé.

\section{RÉSULTATS EXPÉRIMENTAUX ET DISCUSSION}

Les mesures du coefficient global de transfert dans les directions tangentielle et radiale ont été conduites en sys- tème fermé pour trois épaisseurs $(1,2$ et $4 \mathrm{~mm})$ et différentes humidités absolues du bois $(0,8,5$ et $23 \%)$ relatives au domaine hygroscopique.

À titre d'exemple, nous portons sur la figure 3 les variations des premiers membres des relations (7) et (8) en fonction du temps pour des échantillons de hêtre de $4 \mathrm{~mm}$ découpés normalement aux directions tangentielle et radiale et pour différentes humidités du bois. Conformément à la modélisation, la variation des deux premiers membres en fonction du temps est sensiblement linéaire. Les premiers membres ne sont pas strictement égaux à zéro au temps initial car les valeurs des concentrations initiales $C_{1}(0)$ et $C_{2}(0)$ varient légèrement d'une expérience à une autre, les débits d'hélium et d'azote initiaux pouvant être différents de $\pm 10 \%$. Rappelons que chaque point des figures 3 correspond à une expérience complète partant d'une composition à l'instant initial et correspondant à une mesure des concentrations (compartiment 1 ou 2) à un temps choisi par rapport au temps origine.

Pour vérifier les relations (7) et (8), les premiers membres sont estimés avec des valeurs moyennes des concentrations initiales sur l'ensemble des mesures expérimentales effectuées sur un échantillon. C'est la raison pour laquelle la valeur initiale peut être légèrement positive ou négative. Les figures $3 a, b, c$ mettent en évidence les deux points suivants. Les pentes déduites (donc les coefficients de transfert globaux) des deux relations (7) et (8) sont voisines ce qui valide les mesures expérimentales effectuées. De plus, elles sont pratiquement indépendantes de l'état hygroscopique du bois. Ces observations ont été constatées également sur les deux autres épaisseurs.

Désormais, si on porte pour les deux directions étudiées les valeurs de $1 / K S$ en fonction de $e$ (figures 4 et $5)$, les trois épaisseurs ne sont pas suffisantes pour valider une relation linéaire. Toutefois, il est possible d'avoir une estimation de la pente en accordant aux épaisseurs de 2 et $4 \mathrm{~mm}$ une plus grande précision. En effet, pour l'épaisseur de $1 \mathrm{~mm}$ l'influence de la convection même faible peut perturber le transport diffusionnel.

Les droites tracées conduisent à une résistance externe voisine de $2 \times 10^{7} \mathrm{~m}^{-3} \mathrm{~s}^{-1}$ et les valeurs des coefficients de diffusion de l'inerte déduites des pentes sont telles que :

$$
\begin{aligned}
& D_{\mathrm{TI}}=(5,2 \pm 1,6) \times 10^{-8} \mathrm{~m}^{2} \mathrm{~s}^{-1} \\
& D_{\mathrm{RI}}=(8,5 \pm 1,3) \times 10^{-8} \mathrm{~m}^{2} \mathrm{~s}^{-1} .
\end{aligned}
$$

Les deux coefficients sont du même ordre de grandeur. Toutefois la valeur plus élevée du coefficient de diffusion dans la direction radiale peut être attribuée aux rayons ligneux qui n'existent pas dans la direction tangentielle. 

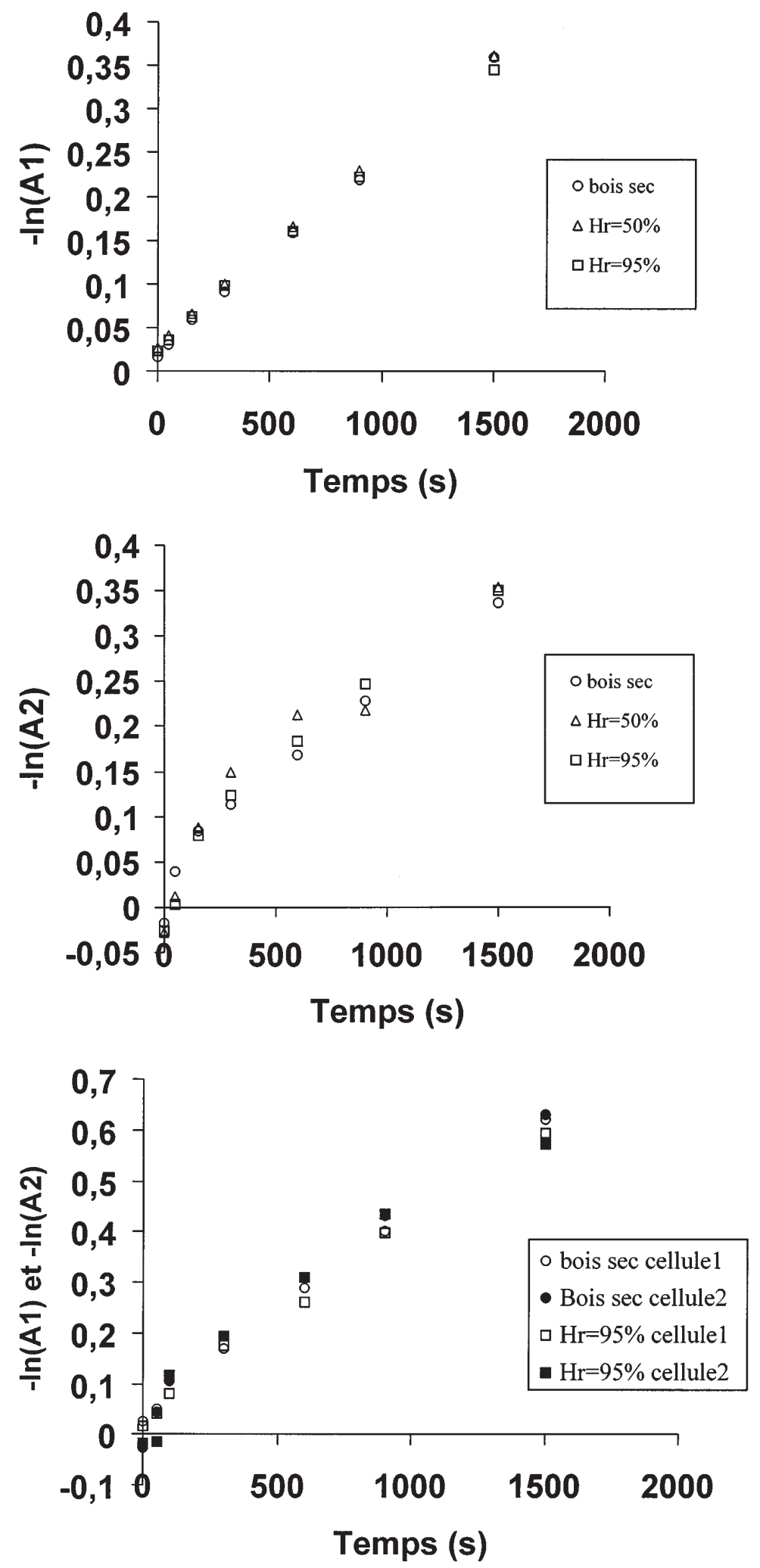

Figure 3a. Exploitation des mesures de concentration dans la cellule 1 en fonction du temps et de l'humidité pour un échantillon de $4 \mathrm{~mm}$ dans la direction tangentielle.

Figure 3b. Exploitation des mesures de concentration dans la cellule 2 en fonction du temps et de l'humidité pour un échantillon de $4 \mathrm{~mm}$ dans la direction tangentielle.

Figure 3c. Exploitation des mesures de concentrations dans les deux cellules en fonction du temps et de l'humidité pour un échantillon de $4 \mathrm{~mm}$ dans la direction radiale. 

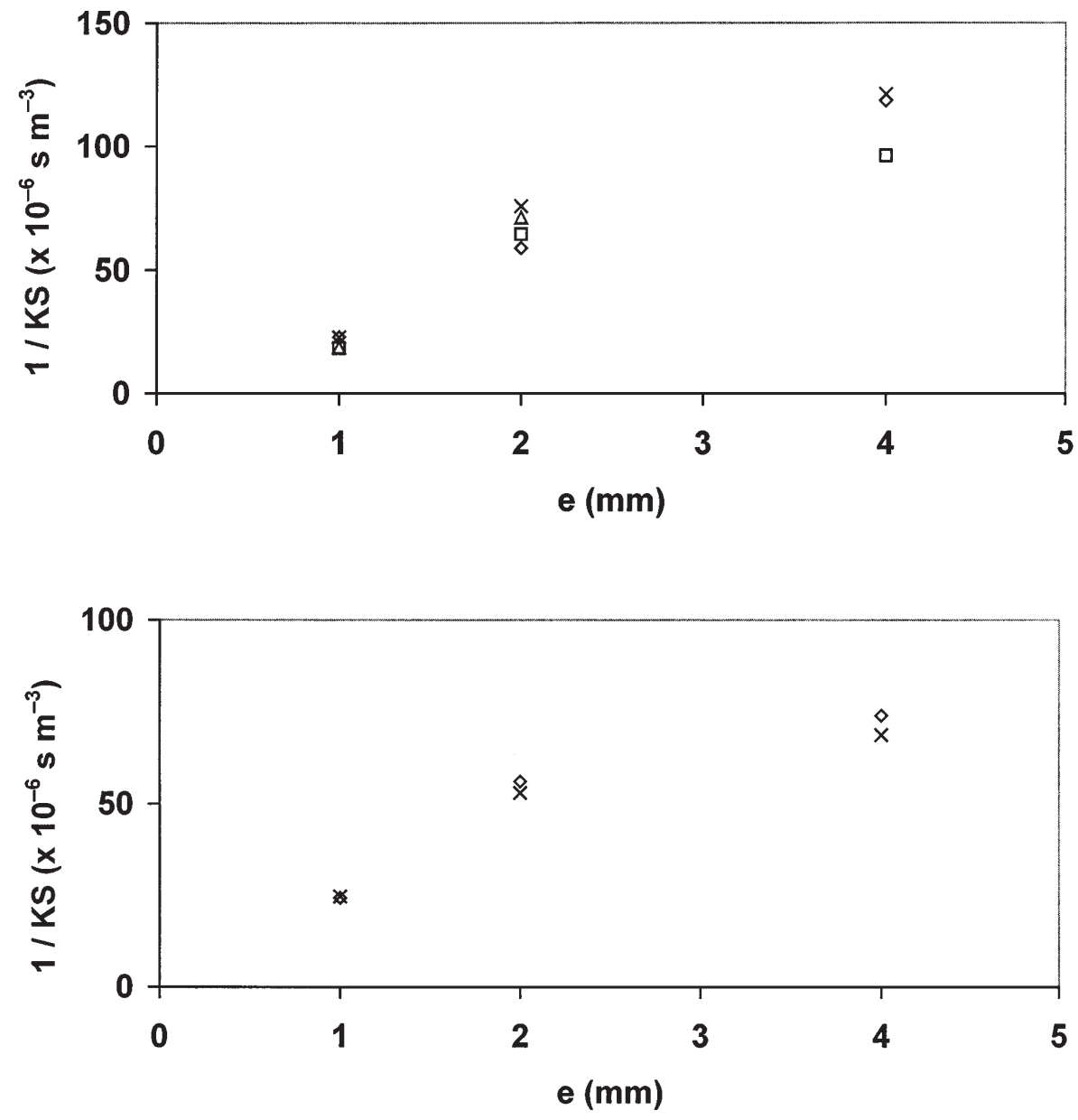

Figure 4. Évolution de $1 / K S$ en fonction de l'épaisseur dans le cas des mesures tangentielles.
A $30^{\circ} \mathrm{C}$, la pression de vapeur de l'eau est négligeable devant celle de l'azote ou de l'hélium. Dans ces conditions, le coefficient de diffusion moléculaire de l'hélium dans le mélange azote-vapeur d'eau est égal au coefficient de diffusion moléculaire de l'hélium dans l'azote. En utilisant la relation de Fuller et coll. [9], à $30{ }^{\circ} \mathrm{C}$ on obtient :

$$
D_{\mathrm{He}-\mathrm{N} 2}=7,32 \times 10^{-5} \mathrm{~m}^{2} \mathrm{~s}^{-1}
$$

De même le coefficient de diffusion de la vapeur d'eau dans de l'air à $30{ }^{\circ} \mathrm{C}$ est tel que :

$$
D_{\text {eau-air }}=2,69 \times 10^{-5} \mathrm{~m}^{2} \mathrm{~s}^{-1} \text {. }
$$

Par suite en utilisant la relation (4), on obtient pour la vapeur d'eau :

$$
\begin{aligned}
& D_{\mathrm{T}}=1,91 \times 10^{-8} \mathrm{~m}^{2} \mathrm{~s}^{-1} \\
& D_{\mathrm{R}}=3,12 \times 10^{-8} \mathrm{~m}^{2} \mathrm{~s}^{-1} .
\end{aligned}
$$

Dans le tableau I, nous avons rassemblé des valeurs de coefficient de diffusion mesurés par différents auteurs par des méthodes stationnaires et transitoires sur différentes essences. Les directions des mesures ne sont généralement pas précisées. Nos valeurs correspondant à des coefficients de diffusion en phase gazeuse, les mesures stationnaires sont plus représentatives de nos déterminations. Ceci est sensiblement vérifié dans la mesure où nos valeurs se situent entre $10^{-8}$ et $10^{-7} \mathrm{~m}^{2} \mathrm{~s}^{-1}$, intervalle compatible avec les intervalles de variations donnés par Siau [11].

\section{CONCLUSIONS}

Par l'intermédiaire de la mesure des coefficients de diffusion d'un soluté gazeux inerte au sein du bois, nous avons pu estimer les coefficients de diffusion de la vapeur d'eau dans les directions tangentielle et radiale du 
Tableau I. Valeurs des coefficients de diffusion mesurés en régime permanent et transitoire données par différents auteurs pour différentes essences de bois.

\begin{tabular}{|c|c|c|c|c|c|c|c|c|}
\hline \multirow{3}{*}{$\begin{array}{l}\text { ESSENCE } \\
\text { SAPIN }\end{array}$} & \multicolumn{3}{|c|}{ Conditions operatoires } & \multirow{2}{*}{\multicolumn{2}{|c|}{$\begin{array}{l}\text { Coefficients de diffusion } \\
\text { obtenus en régime } \\
\text { permanent }\left(\mathrm{m}^{2} \mathrm{~s}^{-1}\right)\end{array}$}} & \multirow{2}{*}{\multicolumn{2}{|c|}{$\begin{array}{l}\text { Coefficients de diffusion } \\
\text { obtenus en régime } \\
\text { transitoire }\left(\mathrm{m}^{2} \mathrm{~s}^{-1}\right)\end{array}$}} & \multirow{3}{*}{$\begin{array}{c}\begin{array}{c}\text { Direction } \\
\text { de mesure }\end{array} \\
{[4]: \overline{\mathrm{R}+\mathrm{T}}} \\
{[3]: \text { a) R }} \\
\text { b) } \mathrm{L} \\
{[1]: ?}\end{array}$} \\
\hline & $\mathrm{T}\left({ }^{\circ} \mathrm{C}\right)$ & M & $\mathrm{Hr}$ & & & & & \\
\hline & $\begin{array}{c}40 \\
71-93,3\end{array}$ & $\begin{array}{l}15 \\
>\end{array}$ & & $3,24 \times 10^{-10}$ & Choong [4] & $\begin{array}{l}2,82 \times 10^{-10(a)} \\
3,0 \times 10^{-10(b)} \\
4,97 \times 10^{-10}\end{array}$ & $\begin{array}{l}\text { Choong [3] } \\
\text { Bramhall [1] }\end{array}$ & \\
\hline PIN & 25 & $0-7,5$ & 75 & $\begin{array}{l}3,50 \times 10^{-7} \\
8,04 \times 10^{-6} \\
4,88 \times 10^{-7}\end{array}$ & $\begin{array}{l}\text { Perré [8] } \\
\text { (duramen) }\end{array}$ & $2,11 \times 10^{-11}$ & Siau [10] & $\begin{aligned} {[8] } & : \mathrm{T} \\
& : \mathrm{L} \\
& : \mathrm{R} \\
{[10] } & : \overline{\mathrm{R}+\mathrm{T}}\end{aligned}$ \\
\hline PEUPLIER & $\begin{array}{l}25 \\
43 \\
40\end{array}$ & 6-16 & $\begin{array}{l}60 \\
-\end{array}$ & $9,22 \times 10^{-9}$ & Perng [7] & $\begin{array}{l}2,10 \times 10^{-10} \\
2,60 \times 10^{-10}\end{array}$ & $\begin{array}{l}\text { Simpson [12] } \\
\text { Comstock [5] }\end{array}$ & $\begin{array}{l}{[7]: \mathrm{L}} \\
{[12]: \mathrm{R}} \\
{[5]: ?}\end{array}$ \\
\hline CHÊNE & $\begin{array}{l}43 \\
-\end{array}$ & & $\begin{array}{l}15 \\
-\end{array}$ & $\begin{array}{l}1,87 \times 10^{-7} \\
2,82 \times 10^{-6} \\
1,85 \times 10^{-7}\end{array}$ & Perré [8] & $\begin{array}{l}1,14 \times 10^{-10} \\
5,46 \times 10^{-10}\end{array}$ & $\begin{array}{l}\text { Simpson [13] } \\
\text { Chen [2] }\end{array}$ & $\begin{aligned} {[8]: \mathrm{T} } \\
: \mathrm{L} \\
: \mathrm{R} \\
{[13]: \mathrm{R} } \\
{[2]: \mathrm{L} }\end{aligned}$ \\
\hline
\end{tabular}

$\mathrm{R}$ : direction radiale, $\mathrm{T}$ : direction tangentielle, $\mathrm{L}$ : direction longitudinale, $\overline{\mathrm{R}+\mathrm{T}}$ : moyenne des directions radiale et tangentielle.

bois de hêtre. Dans le domaine hygroscopique du bois, ces coefficients de diffusion sont indépendants de l'humidité absolue du bois et le coefficient de diffusion dans la direction radiale est supérieur au coefficient de diffusion dans la direction tangentielle.

Il nous faut à présent mesurer le coefficient de diffusion de la vapeur d'eau dans la direction axiale et vérifier si la modélisation proposée permet de bien représenter les phénomènes d'adsorption et de désorption isotherme de la vapeur d'eau dans un échantillon de bois. Enfin, pour appliquer ces valeurs à une opération de séchage, il nous faudra préciser la variation des coefficients de diffusion en fonction de la température. Tels sont les objectifs que nous proposons de réaliser dans la suite de ce travail de recherche.

\section{RÉFÉRENCES}

[1] Bramhall G., Mathematical model of lumber drying, Wood Sci. 12 (1979) 14-21.

[2] Chen Y., Choong E.T., Wetzel D.M., Optimum average diffusion coefficient: an objective index in description of wood drying data, Wood Fiber Sci. 26 (1994) 412-420.

[3] Choong E.T., Movement of water through a softwood in the hygroscopic range, For. Prod. J. 13 (1963) 489-498.
[4] Choong E.T., Diffusion coefficients of softwood by steady state and theoretical methods, For. Prod. J. 15 (1965) 21-27.

[5] Comstock G.L., Moisture diffusion coefficients in wood calculated from adsorption, desorption and steady state data, For. Prod. J. 13 (1963) 97-103.

[6] Liu J.Y., A new method for separating diffusion coefficient and surface emission coefficient, Wood Fiber Sci. 21 (1989) 133-141.

[7] Perng W.R., Brebner K.I., Schneider M.H., Aspen wood anatomy and fluid transport, Wood Fiber Sci. 17 (1985) 281-289.

[8] Perré P., Zohoun S., Brandão A., Détermination de la diffusivité massique en régime permanent - Résultats d'une campagne de mesure sur différentes essences tempérées et tropicales, $4^{\mathrm{e}}$ colloque sciences et industries du bois, Nancy, septembre 1996.

[9] Reid R.C., Prausnitz J.M., Sherwood T.K., The properties of gases and liquids, Third edition, The Mac Graw Hill, New-York 1977, pp. 544-601.

[10] Siau J.F., Non isothermal moisture movement in wood, Wood Sci. 13 (1980) 11-13.

[11] Siau J.F., Wood influence of water on physical properties, Virginia Polytechnic Institute, 1995.

[12] Simpson W.T., Liu J.Y., Dependence of the water diffusion coefficient of aspen (Populus spec.) on moisture content, Wood Sci. Technol. 26 (1991) 9-21.

[13] Simpson W.T., Determination and use of moisture diffusion coefficient to characterize drying of northern red oak, Wood Sci. Technol. 27 (1993) 409-420. 